\title{
Complicações do Pneumotórax em pacientes idosos com Doença Pulmonar Obstrutiva Crônica (DPOC)
}

\author{
Pneumothorax complications in elderly patients with Chronic Obstructive Pulmonary \\ Disease (COPD)
}

\section{Complicaciones del neumotórax en pacientes ancianos con Enfermedad Pulmonar Obstructiva Crónica (EPOC)}

Marcos Filipe Chaparoni de Freitas Silva ${ }^{1 *}$, Anderson Poubel Batista ${ }^{2}$, Clara Liboni Torres Penedo ${ }^{3}$, Débora Angélica Rocha da Cunha Ferreira ${ }^{4}$, Edílio Póvoa Lemes Neto ${ }^{5}$, Geovana de Figueiredo Melo $^{6}$, Igor Reggiani Gomes ${ }^{7}$, Leandro Cesar Nogueira Almeida ${ }^{7}$, Stefanie Flach Zanatta ${ }^{8}$, Renan Guimarães Assunção Campos ${ }^{9}$.

\section{RESUMO}

Objetivo: Compreender as complicações relacionadas entre o pneumotórax e os pacientes idosos com DPOC, bem como, as abordagens terapêuticas e a importância do acompanhamento individualizado desse grupo de pacientes. Revisão bibliográfica: $O$ pneumotórax é uma urgência médica definida pela apresentação de ar na cavidade pleural. É classificado como espontâneo ou não espontâneo originado traumaticamente. O pneumotórax espontâneo é subdividido em primário, quando há ausência de doenças pulmonares subjacentes e secundárias, caracterizado pela presença de patologias pulmonares associadas como a DPOC. A DPOC surge como condição prévia para complicações em pacientes idosos com pneumotórax espontâneo secundário. Dentre as complicações do pneumotórax em pacientes idosos com DPOC, temos a piora nas funções cardiopulmonares, insuficiência respiratória crônica, longos tratamentos com oxigenoterapia e até o óbito. Diante da sensibilidade desse quadro é necessária uma avaliação adequada e individualizada desse pneumotórax, principalmente porque a grande maioria dos pacientes com DPOC são idosos. Considerações finais: Assim, para evitar as complicações graves nesse grupo específico, é necessária a reavaliação e rastreamento da DPOC nessa população, a fim de que o tratamento seja realizado o mais precoce, adequado e individualizado possível, evitando, desta forma, não só o desenvolvimento do pneumotórax, mas, também, das complicações mais graves e fatais.

Palavras-chave: Complicações do pneumotórax, Idosos, Doença pulmonar obstrutiva crônica.

\section{ABSTRACT}

Objective: To understand the complications related between pneumothorax and elderly patients with COPD, as well as, therapeutic approaches and the importance of individualized monitoring of this group of patients. Bibliographic review: Pneumothorax is a medical emergency defined by the presence of air in the pleural cavity. It is classified as spontaneous or non-spontaneous originating from trauma. Spontaneous pneumothorax is subdivided into primary, when there is no underlying lung disease, and secondary,

\footnotetext{
${ }^{1}$ Centro Universitário do Planalto Central Apparecido dos Santos (UNICEPLAC), Brasília - DF.

*E-mail: mchaparoni@gmail.com

${ }^{2}$ Faculdade Metropolitana São Carlos (FAMESC), Bom Jesus do Itabapoana - RJ.

${ }^{3}$ Centro Universitário Municipal de Franca (Uni-FACEF), Franca - SP.

${ }^{4}$ Faculdade de Medicina de Petrópolis (FMP/UNIFASE), Petrópolis - RJ.

${ }^{5}$ Faculdade Presidente Antônio Carlos (ITPAC), Porto Nacional - TO.

${ }^{6}$ Universidade Nilton Lins (UNINILTONLINS), Manaus - AM.

${ }^{7}$ Faculdade de Medicina de Barbacena (FAME), Barbacena - MG.

8 Universidade Federal de Pelotas (UFPel), Pelotas - RS.

${ }^{9}$ Centro universitário de patos de Minas (UNIPAM), Patos de Minas - MG.
} 
characterized by the presence of associated pulmonary pathologies such as COPD. COPD appears as a precondition for complications in elderly patients with secondary spontaneous pneumothorax. Among the complications of pneumothorax in elderly patients with COPD, there is a worsening in cardiopulmonary functions, chronic respiratory failure, long treatments with oxygen therapy and even death. Given the sensitivity of this condition, an adequate and individualized assessment of this pneumothorax is necessary, mainly because the majority of patients with COPD are elderly. Final considerations: So, to avoid serious complications in this group, it is necessary to reassess and monitor COPD in this population, so that the treatment is carried out as early, adequate and individualized as possible, avoiding not only the development of pneumothorax, but also the most serious and fatal complications.

Key words: Pneumothorax complications, Elderly, Pulmonary disease chronic obstructive.

\section{RESUMEN}

Objetivo: Comprender las complicaciones relacionadas entre el neumotórax y los pacientes ancianos con APOC, así como los enfoques terapéuticos y la importancia del seguimiento individualizado de este grupo de pacientes. Revisión bibliográfica: El neumotórax es una urgencia médica definida por la presencia de aire en la cavidad pleural. Se clasifica como espontáneo o no espontáneo originado por un trauma. El neumotórax espontáneo se subdivide en primario, sin enfermedad pulmonar subyacente, y secundario, caracterizado por la presencia de patologías pulmonares asociadas como la APOC. APOC aparece como una condición previa para las complicaciones en pacientes ancianos con neumotórax espontáneo secundario. Entre las complicaciones del neumotórax en pacientes ancianos con APOC, tenemos una empeora de las funciones cardiopulmonares, insuficiencia respiratoria crónica, tratamientos prolongados con oxigenoterapia e incluso la muerte. Dada la sensibilidad de esta patología, es necesaria una valoración adecuada e individualizada de este neumotórax, principalmente porque la mayoría de los pacientes con APOC son ancianos. Consideraciones finales: Así, para evitar complicaciones graves en este grupo específico, es necesario reevaluar y monitorear la APOC en esta población, para que el tratamiento se realice lo más temprano, adecuado e individualizado, evitando así no solo el neumotórax, sino también las complicaciones más graves $y$ fatales.

Palabras clave: Complicaciones del neumotórax, Anciano, Enfermedad pulmonar obstructiva crónica.

\section{INTRODUÇÃO}

O pneumotórax pode ser definido como um distúrbio pulmonar que se manifesta por meio do acúmulo ou presença de ar no espaço pleural e que pode resultar em colapso parcial ou total da capacidade pulmonar. De acordo com a sua etiologia, pode ser classificado em espontâneo ou não espontâneo, sendo este, ocasionado por trauma. O pneumotórax espontâneo é subdividido em primário, no qual, há a ausência de doenças pulmonares subjacentes, e secundário, quando há patologias pulmonares associadas (ONUKI T, et al., 2017).

Segundo Rodriguez MEG, et al. (2019), o pneumotórax espontâneo secundário pode ser desencadeado devido a diversas patologias, como fibrose cística e asma, entretanto, a Doença Pulmonar Obstrutiva Crônica (DPOC) é a principal causa, a qual tem maior prevalência em pacientes idosos, ou seja, pessoas com 60 anos de idade ou mais. Nesse sentido, a DPOC é descrita como condição prévia para complicações em pacientes idosos com pneumotórax espontâneo secundário.

Além disso, deve-se avaliar alguns fatores de risco, como presença de doenças cardiovasculares, câncer de pulmão e outras neoplasias nos pacientes idosos acometidos por doenças pulmonares, por exemplo, a DPOC que apresentam o quadro de pneumotórax espontâneo secundário, para então determinar o manejo desses pacientes (ONUKI T, et al., 2017).

O tratamento deve ser definido como cirúrgico ou não, se baseando no conjunto de possíveis complicações que poderão ser desencadeadas por: idade, presença de associação de doenças de base, assim como o 
tempo e o tipo da possível cirurgia. Por isso, o tratamento precoce é um fator determinante para um resultado bem-sucedido do pneumotórax espontâneo secundário, e que sua diversidade de sintomas demanda um manejo clínico minucioso que pode interferir diretamente no tratamento da doença. As modalidades de tratamento dependem do quadro de cada paciente, no caso de apresentar-se como um quadro leve, realizase drenagem torácica, já nos casos recorrentes, houve a necessidade de intervenção cirúrgica (ICHINOSE J, et al., 2015; KIM D, et al., 2019; ONUKI T, et al., 2017).

Nesse sentido, também deve-se destacar que o perfil de maior prevalência do pneumotórax espontâneo secundário, manifesta-se em homens, idosos, com enfermidades associadas e tendem a ter sintomas mais intensos se comparados a pacientes com pneumotórax espontâneo primário (SCHNELL J, et al., 2017; BINTCLIFFE OJ, et al., 2015; HALLIFAX RJ, et al., 2018; KIM D, et al., 2019).

O pneumotórax tem idade de distribuição bimodal, considerado como o primeiro pico em jovens e o segundo pico em pessoas com 50 anos ou mais (BINTCLIFFE OJ, et al., 2015; HALLIFAX, RJ, et al., 2018; TAKAHASHI F, et al., 2020). No Japão, 60 a $80 \%$ dos casos de pneumotórax espontâneo secundário tem relação com a DPOC ou enfisema pulmonar, embora esses números possam ser diferentes dependendo do país, em pacientes com DPOC, a incidência desse evento é de aproximadamente 26/100.000 anualmente, sendo a incidência na população de modo geral de 6 a 28/100.000 (AKCAM TI, et al., 2017; SEVINC S, et al., 2015; TAKAHASHI F, et al., 2020).

A recorrência desse evento, depende do tratamento utilizado, sendo maior e com altas taxas de hospitalização no pneumotórax espontâneo secundário em comparação com o pneumotórax espontâneo primário (AKCAM TI, et al., 2017; SAITO Y, et al., 2017). Conforme Hallifax RJ, et al. (2018), segundo dados compostos a partir das bases nacionais mais atualizadas da Inglaterra, 60,8\% dos pacientes com pneumotórax espontâneo concomitantemente portavam uma doença pulmonar crônica (sendo por esse fato considerado um pneumotórax espontâneo secundário).

As complicações do pneumotórax em pacientes idosos com DPOC podem ser diversas, como piora nas funções cardiopulmonares, insuficiência respiratória crônica e a introdução ou incrementação de longos tratamentos com oxigenoterapia, seja em seus domicílios ou nos hospitais, além dos casos anteriormente citados relacionados à recorrência do pneumotórax, podendo ocorrer até mesmo a morte desses pacientes (LI H, et al., 2020; SAITO Y, et al., 2017; TAKAHASHI F, et al., 2020). Contudo, é importante uma avaliação adequada em pacientes com pneumotórax e DPOC, principalmente idosos, os quais são a maioria dos pacientes portadores de DPOC, com o intuito de compreender as complicações relacionadas.

Diante disso, o presente artigo de revisão narrativa teve como objetivo reconhecer, aprofundar e transcorrer sobre as complicações do pneumotórax em pacientes idosos com DPOC, a fim de evitar agravos decorrente do pneumotórax.

\section{REVISÃO BIBLIOGRÁFICA}

Desde o início do século 20, tem sido consagrada a classificação do pneumotórax como de origem primária e de origem secundária. Essa classificação foi necessária devido às comorbidades de grande prevalência epidemiológica dessa época, como a Tuberculose. Assim, temos a primeira descrição por Kjærgaard, em 1932, como pneumotórax primário ou "pneumotórax simples", no qual pacientes não apresentavam comorbidades pulmonares, e pneumotórax secundário, na época denominado como "pneumotórax secundária à doença tuberculosa". Tal definição foi de suma importância devido a evitar causas não associadas à tuberculose de pneumotórax, evitando isolamento desses indivíduos em sanatórios (HALLIFAX RJ, et al., 2019).

Assim, atualmente, o pneumotórax é classificado como um distúrbio torácico que se manifesta como coleta anormal de ar, podendo ser causado por lesões torácicas contundentes ou penetrantes, procedimentos médicos ou danos de doenças pulmonares subjacentes. O pneumotórax espontâneo se desenvolve na ausência de trauma, podendo ser classificado como primário e secundário. Enquanto o pneumotórax primário afeta pacientes sem distúrbios pulmonares clinicamente aparentes, o secundário envolve uma doença 
pulmonar subjacente e a que é mais frequentemente associada a este tipo é a DPOC, a qual afeta, em suma, idosos (ONUKI T, et al., 2017).

Desde então, com a evolução da medicina moderna, nosso entendimento de várias patologias se expandiu, e assim a ciência do pneumotórax evoluiu em espectro. Várias enfermidades foram incluídas em ambas as classificações, pneumotórax primário e secundário, tendo este último evoluído principalmente numa gama de doenças como DPOC, neoplasias, doenças pulmonares intersticiais, AIDS, pneumoconioses, entre outros. Hodierno, dados epidemiológicos demonstram como prevalência a Doença Pulmonar Obstrutiva Crônica, enfermidade predominante em idosos, correspondendo 60 a $80 \%$ de todos os casos de pneumotórax secundários no Japão (TAKAHASHI F, et al., 2020).

Independentemente do fator predisponente, ou seja, a doença de base, esses pacientes já possuem a função pulmonar comprometida, sendo o pneumotórax secundário uma doença potencialmente fatal e que necessita de tratamento eminente (ONUKI T, et al., 2017). A origem dessa patologia é explicada pelo mecanismo fisiopatológico da DPOC, no qual o aumento anormal e permanente dos espaços aéreos distais aos bronquíolos terminais acompanha a destruição das paredes dos espaços aéreos. Tais deformidades acarretam em blebs ou bolhas apicais que se rompem levando ao extravasamento de ar para cavidade pleural (NOPPEN M, et al., 2008).

As análises retrospectivas de admissões hospitalares por Pneumotórax Espontâneo no Japão, Alemanha, Coreia e Inglaterra afirmam que há dois picos de incidência do Pneumotórax Espontâneo: entre pacientes jovens, sem comorbidades e tipo pneumotórax espontâneo primário e pacientes idosos, a partir dos 60 anos, com comorbidades e tipo Pneumotórax espontâneo secundário. Ressalta-se que o pneumotórax espontâneo primário é o mais comum nessas populações (ONUKI T, et al., 2015; SCHNELL J, et al., 2017; KIM D, et al., 2019; HALLIFAX RJ, et al., 2018; TAKAHASHI F, et al., 2020).

Houve uma maior proporção de homens com o diagnóstico de pneumotórax espontâneo em relação às mulheres nos estudos de Schenell J, et al. (2017) na Alemanha, de Kim D, et al. (2019) na Coreia, e Hallifax RJ, et al. (2018) na Inglaterra, mas não foi observado diferença significativa entre os sexos no Japão por Onuki T, et al. (2015). Dentre as comorbidades apresentadas no Japão, Alemanha, Coreia e Inglaterra, a DPOC é a mais frequente doença associada ao pneumotórax espontâneo (ONUKI T, et al., 2015; SCHNELL J, et al., 2017; KIM D, et al., 2019; HALLIFAX RJ, et al., 2018).

Na Alemanha, a frequência anual de pacientes internados por pneumotórax espontâneo, entre 2011 a 2015, foi de 14,3/100.000. Dentre os pacientes que tiveram um Pneumotórax Espontâneo Secundário, 16,2\% possuíam DPOC (comorbidade mais observada) e a média de idade foi 70-80 anos (SCHNELL J, et al., 2017).

A prevalência anual de pneumotórax espontâneo que levaram a hospitalizações na Coreia variou entre 18-36/100.000 indivíduos. Kim D, et al. (2019), afirma que dos pacientes com mais de 65 anos (497 indivíduos), cerca de $57,7 \%$ (287 indivíduos) possuíam DPOC, configurando como a comorbidade mais prevalente. Além disso, de 3.003 indivíduos com a sua primeira hospitalização, 1.683 pacientes foram hospitalizados novamente, $60,7 \%$ nos primeiros 6 meses e $71,4 \%$ em um ano. Os fatores de risco envolvidos na uma reinternação ainda necessitam de maiores elucidações.

$\mathrm{Na}$ Inglaterra, Hallifax RJ, et al. (2018) afirma que no ano de 2015 das internações hospitalares por pneumotórax espontâneo secundário em maiores de 65 anos cerca de $87,6 \%$ dos homens e $79,2 \%$ das mulheres possuíam doença pulmonar obstrutiva crônica ou enfisema. Com relação a recorrência de um pneumotórax espontâneo, Hallifax RJ, et al. (2018) sugere que há uma maior taxa entre os pacientes com alguma doença pulmonar em relação aos que não possuem.

Segundo Filho LOA, et al. (2006), o tratamento do pneumotórax pode variar, de acordo três aspectos: condições clínicas do paciente, etiologia, magnitude. Com isso, pode haver desde tratamentos mais conservadores, até tratamentos cirúrgicos como a toracotomia com ressecção pulmonar e pleurectomia. Portanto, uma anamnese e exame físico detalhados são de extrema relevância para direcionar o tratamento desse paciente. 
Segundo Barbera AR e Jones MP (2016), em idosos a sintomatologia clássica do pneumotórax em idosos envolve dor, taquipneia, taquicardia, hipotensão e hipóxia, além de achados físicos como murmúrio vesicular focal ou unilateralmente diminuídos e percussão pulmonar alterada em ambos os lobos. Os autores destacam também a importância dos exames complementares no diagnóstico e escolha da intervenção, tendo em vista que achados característicos são encontrados no raio-x e a Tomografia Computadorizada pode ser essencial para determinar a causa do evento, caso não tenham feito descobertas relevantes apenas com a radiografia, como nos casos de doenças parenquimatosas.

Em um pneumotórax espontâneo secundário, necessita-se de internação e de drenagem torácica com dreno tubular como tratamento inicial de escolha na maioria dos casos, salvo em pneumotórax pequenos e oligo ou assintomáticos. A maioria dos casos de pneumotórax espontâneo secundário, no entanto, irá necessitar de toracostomia com drenagem fechada sob selo d'água vulnerabilizada (BARBOSA ATF, et al., 2017).

Há também a alternativa de escolha terapêutica inicial com aspiração por agulha, usando jelcos $14 \mathrm{G}$ ou $16 \mathrm{G}$, drenando um volume total de até 2,5L. Tal terapêutica, apesar de apresentar taxas de sucesso que variam de 48 a $80 \%$ segundo Halifax RJ, et al. (2019), apresenta maiores índices de falha terapêutica em comparação à toracostomia com drenagem fechada sob selo d'água. Contudo, sua escolha como terapêutica inicial é benéfica devido a proporcionar menor tempo de hospitalização e menor incidência de complicações relacionadas ao procedimento cirúrgico instituído inicialmente.

Há casos que apesar da aspiração por agulha e subsequente toracostomia com drenagem fechada sob selo d'água apresenta falha terapêutica, na qual é definida por reexpansão pulmonar insuficiente ou perda aérea prolongada. Guidelines recentes sugerem a permanência do dreno de tórax fechado em selo d'água por um tempo mínimo de 3 a 5 dias para avaliar se há falha terapêutica. Caso não haja resolução do pneumotórax com reexpansão pulmonar adequada e ausência de perda aérea nesse tempo, a avaliação do cirurgião torácico faz-se necessária. O especialista diante de tal situação pode optar em submeter o paciente ao procedimento cirúrgico de Toracoscopia Videoassistida (TVA) com objetivo de identificar e grampear áreas de perda aérea e/ou ressecar blebs ou bolhas enfisematosas visíveis na pleura visceral (HALLIFAX RJ, et al., 2019).

É comumente realizado a pleurodese após o tratamento das bolhas enfisematosas e áreas de perda aérea com o objetivo de colabar os folhetos viscerais e parietal pleurais, culminando na sínfise do espaço pleural impossibilitando o acúmulo de ar. A pleurodese pode ser realizada através de técnicas com estímulos físicos ou mecânicos como a abrasão do mesotélio pleural ocasionando atividade inflamatória com proliferação fibroblástica com depósito de colágeno entre os folhetos pleurais possibilitando a sínfise do espaço pleural. Estímulos químicos também podem ser utilizados com o uso de talco, doxiciclina, nitrato de prata ou bleomicina culminando em irritação dos tecidos com o objetivo de ocasionar a pleurodese (HALLIFAX RJ, et al., 2019).

O médico deve considerar não só o tratamento do pneumotórax, como também a estabilização e o tratamento da condição-base do paciente para que ajude no desenvolvimento favorável do quadro clínico mais grave deste paciente (FILHO LOA, et al., 2006). Dessa forma, sendo a DPOC a condição mais associada, como mencionado anteriormente, a intervenção deve ser focada na reabilitação e broncodilatação dos pulmões.

Segundo Filho LOA, et al. (2006), a justificativa para esta conduta mais invasiva está no fato de que pacientes que já possuem uma doença pulmonar de base (provável causadora do pneumotórax secundário, como o DPOC) apresentam uma menor complacência de seus pulmões, e de que muitas vezes um pneumotórax que aparenta ser pequeno já é suficiente para gerar graves consequências, visto que o sistema respiratório desse paciente já se encontra comprometido.

É importante então, notar que, no pneumotórax secundário, a função pulmonar desses pacientes acometidos já está comprometida e, portanto, ao contrário da afecção primária, a secundária frequentemente se apresentará como uma doença potencialmente fatal, exigindo ação imediata (ONUKI T, et al., 2017). 
Associado a esta elevada gravidade, o subdiagnóstico da DPOC como algo muito presente nessa população idosa, devido à ausência da associação clássica dos sintomas respiratórios nos idosos, faz com que essa condição em maiores de 60 anos seja vista como algo muito preocupante e necessitado de reavaliações no que se diz respeito às condutas frente essa população, que se encontra em risco e vulnerabilizada (BARBOSA ATF, et al., 2017).

Como consequência da prevalência do subdiagnóstico, temos o não tratamento, que aumenta a morbidade e riscos de complicações nos idosos, contribuindo então para a ocorrência pneumotórax secundário à DPOC em idosos (BARBOSA ATF, et al., 2017). Portanto, para que se evite a ocorrência de pneumotórax nessa população, torna-se imprescindível um tratamento voltado para a condição de DPOC, de forma mais precocemente possível, para que se evite sua progressão.

A DPOC é considerada um processo inflamatório crônico e progressivo que ocorre nos pulmões, em decorrência da inalação de partículas de gases tóxicos, resultando na obstrução fixa das vias aéreas que tem como consequência direta a limitação do fluxo expiratório, levando ao comprometimento da função intra e extrapulmonar e, embora seja tratável, não é totalmente reversível. Ela pode ser classificada em 4 graus: I) leve, II) moderada, III) grave e IV) muito grave; sendo que as manifestações clínicas aumentam de acordo com o grau da doença (ALMEIDA JTS e SCHNEIDER LF, 2019).

Ainda segundo Almeida JTS e Schneider LF (2019), os sinais clínicos da DPOC ocorrem de forma sistêmica, podendo gerar dispneia (principal sintoma), tosse, expectoração crônica, presença de sibilos em ausculta respiratória e aspecto de tórax de tonel (consequência da hiperinsuflação pulmonar). Outras características incluem a sensação de cansaço a esforços mínimos, fraqueza muscular, pode ainda apresentar comprometimento cardiovascular, perda de peso e predisposição à associação a outras doenças, se não tratado (ZONZIN, et al., 2017).

O tratamento da DPOC é voltado para o controle e estabilização do quadro clínico da doença e este ocorre por meio de uma equipe multidisciplinar, composta por médico, nutricionista, psicólogo, fisioterapeuta e dentre outros profissionais que atuam cada um com sua especialidade buscando a melhoria dos sintomas e bemestar ao paciente (ALMEIDA JTS e SCHNEIDER LF, 2019). Portanto, é importante deixar claro ao paciente que sua doença é crônica, sem perspectivas de cura, apenas de controle de seus sintomas e prevenção de outras comorbidades.

A medida conservadora abrange a atuação do fisioterapeuta, que se compõe de suma importância para esses pacientes, para que haja uma melhora significativa da função pulmonar e, consequentemente, da qualidade de vida e independência do paciente (ALMEIDA JTS e SCHNEIDER LF, 2019). Segundo o autor, a fisioterapia atuará com diversas técnicas de manobras buscando promover a higiene brônquica, melhorar a capacidade funcional, reduzir o ciclo de fadiga e dispneia, técnicas que auxiliem na desinsuflação pulmonar, melhoria do condicionamento físico e força dos músculos respiratórios e musculatura periférica, objetivando uma maior independência, autonomia, qualidade de vida e autoestima.

Entre as principais abordagens, têm-se manobras para desobstrução brônquica, atividades físicas coordenadas, ventilação não invasiva, oxigenação domiciliar, além de eletroestimulação neuromuscular caso o paciente não consiga realizar as demais atividades de esforço (DOMINGUES PW, et al., 2010).

Segundo Almeida JTS e Schneider LF (2019), a atuação nutricional também é indicada, para evitar que o paciente fique debilitado ou em sobre peso que compromete tanto à saúde como também dificulta ações de intervenção a doença. O acompanhamento com psicólogo pode ser necessário visto que as principais comorbidades envolvem a predisposição de depressão e ansiedade advinda das debilidades da patologia e também de sua cronicidade.

Além desses tratamentos e dessas intervenções associadas, Almeida JTS e Schneider LF (2019) abordam também o tratamento medicamentoso com broncodilatadores. Todos, em associação, contribuem para evitar a progressão da doença e, assim, suas possíveis complicações como o pneumotórax espontâneo secundário. Entretanto, para que essa progressão seja realmente efetivada, existe ainda o papel do paciente no que se diz respeito ao controle de exposição a fatores de risco. 
É de suma importância prestar informações educativas ao paciente sobre a doença em geral para ele obtenha maior consciência sobre o tratamento, pois apesar de a reabilitação reduzir os sintomas, se não forem associados a inibição dos fatores de risco que exacerbam a doença, a reabilitação não será suficiente para evitar a progressão rápida da DPOC (ALMEIDA JTS e SCHNEIDER LF, 2019).

\section{CONSIDERAÇÕES FINAIS}

Portanto, por intermédio do presente trabalho e objetivando uma conclusão a respeito do desenvolvimento e complicações do pneumotórax secundário em pacientes idosos portadores de DPOC, entende-se que, para que sejam evitadas complicações graves nesse grupo, faz-se necessário uma reavaliação do rastreamento da DPOC nessa população para que o tratamento seja realizado precoce e adequadamente, evitando assim não só o desenvolvimento de um pneumotórax, mas também complicações graves e/ou fatais caso o mesmo seja desenvolvido. Ainda com relação a esse grupo sujeito a reavaliação, torna-se imprescindível salientar que, como já dito anteriormente, idosos, homens e pacientes com patologias associadas estão mais suscetíveis ao pneumotórax espontâneo secundário. Sendo assim, se a condição se desenvolver, é importante salientar que seu manejo é, muitas vezes, um desafio e exige preparo, assertividade e dominância do quadro do paciente pela equipe de saúde que o trata e/ou o acompanha.

\section{REFERÊNCIAS}

1. AKCAM TI, et al. Analysis of the patients with simultaneous bilateral spontaneous pneumothorax. The Clinical Respiratory Journal, 2017; 12(3): 1207-1211.

2. ALMEIDA JTS, SCHNEIDER LF. Importância da atuação fisioterapêutica para manter a qualidade de vida dos pacientes com Doença Pulmonar Obstrutiva Crônica - DPOC. Rev Cient da Fac Educ e Meio Ambiente: Revista da Faculdade de Educação e Meio Ambiente - FAEMA, Ariquemes, 2019; 10(1): 167-176.

3. BARBERA AR, JONES MP. Dyspnea in the Elderly. Emergency Medicine Clinics Of North America, 2016; 34(3): 543558

4. BARBOSA ATF, et al. Fatores associados à Doença Pulmonar Obstrutiva Crônica em idosos. Revista Ciência \& Saúde Coletiva, 2017; 22(1): 63-73.

5. BINTCLIFFE OJ, et al. Spontaneous pneumothorax: time to rethink management? The Lancet Respiratory Medicine, 2015; 3(7): 578-588.

6. DOMINGUES PW, et al. Efeitos da intervenção fisioterapêutica como tratamento complementar em portadores de doenças respiratórias. Revista F@pciência, 2010; 6(2): 9-18.

7. FILHO LOA, et al. Pneumotórax. J Bras Pneumol. 2006; 32: S212-S216.

8. HALLIFAX RJ, et al. Pneumothorax - Time for New Guidelines. Semin Respir Crit Care Med. 2019; 40(3): 314-322.

9. HALLIFAX RJ, et al. Trends in the Incidence and Recurrence of Inpatient-Treated Spontaneous Pneumothorax, 19682016. JAMA, 2018; 320(14): 1471-1480.

10. ICHINOSE J, et al. Results of surgical treatment for secondary spontaneous pneumothorax according to underlying diseases. European Journal Of Cardio-Thoracic Surgery, 2015; 49(4): 1132-1136.

11. KIM D, et al. Epidemiology and medical service use for spontaneous pneumothorax: a 12-year study using Nationwide cohort data in Korea. BMJ Open, 2019; 9(10): e028624.

12. $\mathrm{LI} \mathrm{H}$, et al. TIMP-1 and MMP-9 expressions in COPD patients complicated with spontaneous pneumothorax and their correlations with treatment outcomes. Pak J Med Sci., 2020; 36(2): 192-197.

13. NOPPEN M, et al. Pneumothorax. Respiration, 2008; 76(2): 121-127.

14. ONUKI T, et al. Primary and Secondary Spontaneous Pneumothorax: prevalence, clinical features, and in-hospital mortality. Canadian Respiratory Journal, 2017; 2017: 1-8.

15. RODRIGUEZ MEG, et al. Resultados del tratamiento con pleurostomía en pacientes con neumotórax espontáneo. Rev Cubana Cir, 2019; 58(1): e718.

16. SAITO Y, et al. The outcome and risk factors for recurrence and extended hospitalization of secondary spontaneous pneumothorax. Surgery Today, 2017; 48(3): 320-324.

17. SCHNELL J, et al. Spontaneous pneumothorax - epidemiology and treatment in Germany between 2011 and 2015. Dtsch Arztebl Int 2017; 114: 739-44.

18. SEVINC S, et al. Prolonged air leakage in secondary spontaneous pneumothorax: is proportion of emphysema important? The Clinical Respiratory Journal, 2015; 11(6): 833-838.

19. TAKAHASHI F, et al. Etiology and prognosis of spontaneous pneumothorax in the elderly. Etiology and prognosis of spontaneous pneumothorax in the elderly. Geriatr. Gerontol. Int., 2020; 20(10): 878-884.

20. ZONZIN, et al. O que é importante para o Diagnóstico da DPOC? Diretoria da Sopterj-Biênio 2015/2017, 2017; 26(1): $5-14$. 